\title{
External model validation of binary clinical risk prediction models in cardiovascular and thoracic surgery
}

\author{
Graeme L. Hickey, PhD, ${ }^{a}$ and Eugene H. Blackstone, $\mathrm{MD}^{\mathrm{b}}$
}

\begin{abstract}
Clinical risk-prediction models serve an important role in healthcare. They are used for clinical decision-making and measuring the performance of healthcare providers. To establish confidence in a model, external model validation is imperative. When designing such an external model validation study, thought must be given to patient selection, risk factor and outcome definitions, missing data, and the transparent reporting of the analysis. In addition, there are a number of statistical methods available for external model validation. Execution of a rigorous external validation study rests in proper study design, application of suitable statistical methods, and transparent reporting. (J Thorac Cardiovasc Surg 2016;152:351-5)
\end{abstract}

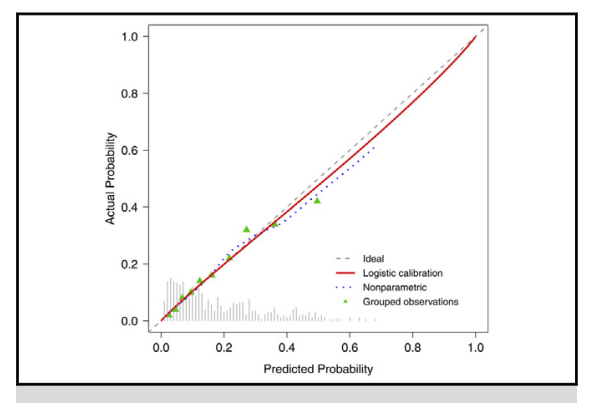

A calibration plot.

Central Message

External validation of binary clinical riskprediction models is vital. We provide strategies for accomplishing this.

\section{Perspective}

The important role of clinical risk-prediction models for clinical decision-making and healthcare provider monitoring requires that they be externally validated. A model that has poor calibration or discrimination can result in misleading conclusions and suboptimal decision-making. This article highlights the key concepts.

See Editorial Commentary page 356
Clinical risk-prediction models (CRPMs; also known as prognostic models or risk score models) serve an important role in healthcare, ${ }^{1}$ particularly for binary adverse events (in-hospital, 30-day, or operative mortality) after cardiac, thoracic, and vascular surgery. These models may be applied to 3 different objectives: (1) to assess patient risk, which surgeons and patients can then factor in to healthcare decisions; (2) to stratify risk, both for clinical decision making and for determination of inclusion criteria in a controlled randomized trial ${ }^{2}$; and (3) to assess and compare healthcare outcomes among providers (benchmarking). The comparison of

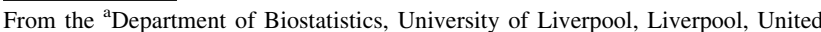
Kingdom and ${ }^{\mathrm{b}} \mathrm{Heart}$ and Vascular Institute, Cleveland Clinic, Cleveland, Ohio.

Received for publication March 1, 2016; revisions received March 22, 2016; accepted for publication April 2, 2016; available ahead of print May 20, 2016.

Address for reprints: Graeme L. Hickey, PhD, Department of Biostatistics, University

of Liverpool, Waterhouse Building (Block F), 1-5 Brownlow St, Liverpool L69

3GL, United Kingdom (E-mail: graeme.hickey@liverpool.ac.uk). $0022-5223 / \$ 36.00$

Copyright (C) 2016 by The American Association for Thoracic Surgery http://dx.doi.org/10.1016/j.jtcvs.2016.04.023
}

observed and expected outcomes, accounting for statistical uncertainty, can identify underperforming healthcare providers for quality improvement interventions.

The wide-ranging importance of CRPMs in the cardiovascular specialty means that stakeholders must have confidence in them. A poorly performing model can lead to suboptimal decision making, misinformed patients, false reassurance of a healthcare provider's performance, or unfair stigmatization of a healthcare provider. Confidence is established by validating the model. ${ }^{4}$

Model validation can be internal, temporal, or external. Internal model validation is one element of CRPM development, usually published alongside the model to confirm that the model performs well for the training data. External validation, which evaluates the generalizability (or transportability) of the model to other groups of patients, is fundamental to demonstrating that a model is appropriate for adoption in clinical practice. ${ }^{4}$ In cardiovascular and thoracic surgery, the majority of CRPMs encountered will 


\section{Abbreviations and Acronyms}

AUROC $=$ area under the receiver operating characteristic curve

$\mathrm{CRPM}=$ clinical risk-prediction model

STS = Society of Thoracic Surgeons

predict binary outcomes, which were created using multivariable regression techniques, in particular logistic regression. Therefore, we focus our discussion here on this area. However, the general principles and need for external validation apply to other outcome types and models, such as time-to-event data, ${ }^{5,6}$ as well as to nonregression techniques, such as machine learning approaches.

\section{MODEL PERFORMANCE CONCEPTS}

Performance of CRPMs is typically assessed based on 2 important features: calibration and discrimination. ${ }^{6}$ Calibration refers to the accuracy of the model for predicting events relative to observed events in groups of patients. For example, if the mean predicted event occurrence is $5 \%$ in a patient group but the observed event occurrence is $10 \%$, then we conclude that the model is not well calibrated because it underpredicts.

Discrimination refers to the ability of a model to distinguish between patients who experienced the event and those who did not. Discrimination is measured using the area under the receiver operating characteristic curve (AUROC), also referred to as the concordance (c)-statistic or $c$-index. ${ }^{5}$ This value has a meaningful interpretation. If we randomly select 2 patients, 1 patient who experienced the event and 1 who did not, then the AUROC is equivalent to the probability that the risk score attributed to the former is greater than that attributed to the latter. An AUROC of 1 indicates perfect classification; a value of 0.5 is equivalent to tossing a fair coin.

Other aspects of performance assessment include clinical usefulness, impact, ${ }^{8}$ and overall performance measures such as the Brier score. ${ }^{9}$

\section{DESIGNING AND REPORTING AN EXTERNAL VALIDATION}

When designing a validation study, thought must be given to various key elements, including selection of patients, risk factor data, missing data, sample size, outcome definitions, study window size, and the transparent reporting of a multivariable prediction model for individual prognosis or diagnosis (TRIPOD).

\section{Selection of Patients}

The selection of patients used to externally validate a CRPM might differ from those used to develop the model. These differences might be temporal or geographical, or related to clinical setting, inclusion or exclusion criteria, definitions, diagnostic techniques, or inherent baseline case mix differences between the 2 populations. It is important to highlight any differences that might affect model transportability between the validation sample and the original study sample, particularly with validation of general all-surgery models (eg, EuroSCORE) within procedural ${ }^{10}$ or operative subgroups. ${ }^{11}$

\section{Risk Factor Data}

It goes without saying that calculating a risk score requires access to all variables that compose the risk score. One potential issue is conflict in variable definitions. For example, a registry that only collects binary data on whether pulmonary artery (PA) systolic pressure is $>60 \mathrm{mmHg}$ (a risk factor in the logistic EuroSCORE model) would not be able to compute the EuroSCORE II risk score, which includes model coefficients for PA systolic pressures of 31 to $55 \mathrm{mmHg}$ and $>55 \mathrm{mmHg}$. This is primarily an issue for retrospective validation studies, because clinical registries can be updated to capture contemporary risk score data.

\section{Missing Data}

One cannot calculate a risk score without access to data for variables that compose the CRPM. If a model contains a risk factor such as preoperative serum creatinine level but these data are sparsely available in the dataset, then in many cases the risk score cannot be calculated. Case-complete analyses-those that delete subjects with missing data for required variables - might lead to bias if those subjects are not representative of the whole population. ${ }^{12}$ In certain cases, reasonable estimates and assumptions can be made based on clinical expertise or additional information in the dataset. A number of variables in Society of Thoracic Surgeons (STS) risk models have coefficients set to 0 for some variables in some models; if one is validating such a model, then missing data for such a variable is of no consequence. Alternatively, statistical imputation or subset analysis techniques might be applied to compensate. ${ }^{13,14}$ If a validation study specifically excludes certain groups of patients (eg, emergency surgery, reoperations, or endocarditis), then imputation of 0 is an accurate and appropriate substitution, but the validation is only partial. In any case, it is always necessary to summarize the frequency of missing data and present methods for managing it and its assumptions.

\section{Sample Size}

Considerations regarding sample size should not be limited to randomized control trials. Single-center validation studies often will have a limited pool of subjects, especially for subgroup analyses, and increasing the sample size will require expanding the study period, which could come at a price (see the comment on calibration drift below). When designing a study, sample size (ie, number of subjects) alone is not enough; one also must consider effective 
sample size (ie, number of events). Relatively little attention has been given to this matter, but some studies have recommended a minimum of 100 events and 100 nonevents for validation studies, and in certain applications, larger effective sizes will be required to obtain adequate power. ${ }^{15,16}$

\section{Outcome Definitions}

Many well-known CRPMs in cardiac surgery, including the logistic EuroSCORE mode $1^{17}$ and STS cardiac surgery risk models, ${ }^{18-20}$ predict early or operative mortality. Operative mortality is generally accepted to mean death within 30 days (or later if the patient has not been discharged within 30 days) ${ }^{21}$; however, other definitions of mortality exist, such as in-hospital mortality. ${ }^{22}$ Two large databases have reported operative mortality of $4.63 \%$ and $3.57 \%$, compared with in-hospital mortality of $4.02 \%$ and $2.94 \%$, respectively. ${ }^{23,24}$ In both cases, in-hospital mortality was approximately $0.6 \%$ lower. In-hospital mortality is generally easier to measure robustly, whereas 30-day mortality requires postdischarge follow-up for most patients. ${ }^{25}$ Therefore, it is common to see models validated against in-hospital mortality. In this example, we would expect the model to overpredict mortality relative to the observed data. It is reasonable to assess the model performance for this similar endpoint; however, this subtlety should be borne in mind when designing a study, particularly if the objective of the study is to compare models with different outcome definitions. Similar considerations apply to cases where the definition of a major postoperative complication used for model development differs from that in the validation dataset.

\section{Study Window Size}

One simple way to increase sample size in a validation study is to expand the study window. Validation of a CRPM over a substantially wide period can introduce a number of complexities, however. One potential issue is calibration drift. ${ }^{26,27}$ Multiple studies have demonstrated a decreasing ratio of observed mortality to mean logistic EuroSCORE with time. Changing risk profiles, other variables influencing mortality, and changes in the association of risk factors with outcomes can all contribute to this phenomenon. This situation prompted the introduction of the EuroSCORE II model ${ }^{23}$ and the series of contemporary STS models. ${ }^{18-20}$ Researchers should be aware of this, particularly when validating cardiac surgery CRPMs.

\section{TRIPOD Statement}

In recent years, reporting of biomedical research has been improved with guidelines such as the CONSORT statement ${ }^{28}$ for randomized trials and the PRISMA statement ${ }^{29}$ for systematic reviews and meta-analyses. Prompted by evidence of poor quality reporting in the CRPM literature, the recently published TRIPOD statement describes reporting guidelines for studies developing, validating, or updating a prediction model. ${ }^{30}$ We strongly encourage researchers to follow these guidelines and make use of the checklist for validating models. Examples of good practice and additional details have been published previously. ${ }^{31}$

\section{METHODS FOR ASSESSING CALIBRATION Hosmer-Lemeshow Test}

The Hosmer-Lemeshow test is a frequently reported statistical test for assessing calibration in CRPMs. This test has several drawbacks, however. ${ }^{31-35}$ First, it is not easily interpreted; that is, it does not provide a measure of the magnitude of any miscalibration. Second, for slight deviations in calibration, the test is sensitive to sample size. Third, the classical version of the test is dependent on arbitrary groupings of patients. In some cases, the Hosmer-Lemeshow test remains a useful adjunct statistic, but should be included only as part of a more comprehensive assessment. Typically, the Hosmer-Lemeshow test refers to a test based on 10 groups composed by deciles of risk; however, one should be aware that there are variations of the test with regard to groupings (quantiles vs fixed cutpoints), number of groups $(g)$, degrees of freedom of the $\chi^{2}$ statistic ( $g-2$ for internal vs $g$ for external validation), and software implementation. ${ }^{35,36}$ Although $g$ is typically selected to be 10 , one must ensure that the cell counts are sufficient to justify the distributional approximation. Including a table of observed and expected events by binning group provides a useful summary, and allows for inspection of each term for fit, as recommended by Hosmer and Lemeshow (p. 188). ${ }^{36}$

\section{Calibration Plot}

If a standard Hosmer-Lemeshow test is performed, then a simple graph - the calibration plot-is a straightforward next step (Figure 1). ${ }^{4}$ Within each of the $g$ groups, observed events are plotted against expected events. If the model is well calibrated, then these points should be close to the 45-degree line. The calibration plot can be augmented by overlaying a nonparametric smoothing curve (eg, loess) through the observed and predicted data ${ }^{37}$ or a calibration curve. ${ }^{38}$ In contrast to the Hosmer-Lemeshow test and basic calibration plot, these additional fits are not dependent on arbitrary groupings.

\section{Calibration Curves}

Cox's calibration regression fits a logistic regression between the observed event and the log-odds transformed predicted values. ${ }^{39} \mathrm{~A}$ perfectly calibrated CRPM (deriving from a logistic regression model) yields an intercept of 0 and a slope of 1 . These fitted regression models can be superimposed onto a calibration plot, giving an alternative graphical description of the miscalibration. Along with quantifying the degree of miscalibration, one also can simultaneously test whether the estimated parameters reject the null hypothesis of calibration. There are other related null hypotheses that can be tested for assessing calibration, as described by Steyerberg (p. 274).

\section{Other Tests}

Although the Hosmer-Lemeshow test is ubiquitous in the biomedical CRPM literature, researchers can use a wide variety of statistical tests to assess model validation, such as the aforementioned calibration curve tests, the Spiegelhalter $Z$-test for calibration accuracy, ${ }^{40}$ and methods proposed by Stallard. ${ }^{41}$ Most of these can be calculated using routine software packages. ${ }^{6,38}$ There is no omnibus test of calibration; each approach has different merits and limitations. Therefore, it is important that researchers use a broad repertoire of methods to address the study questions. 


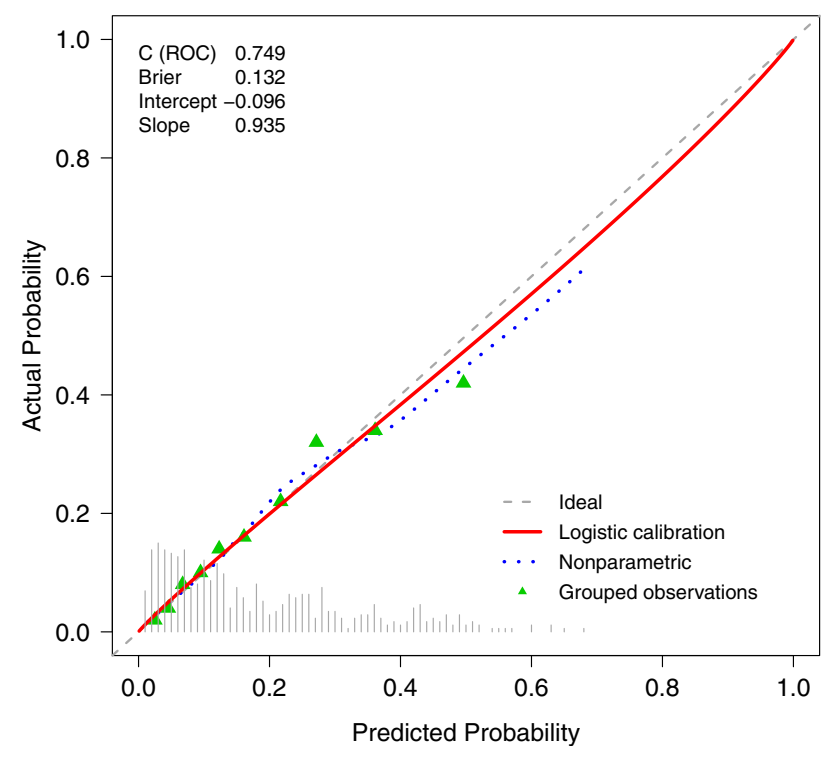

FIGURE 1. Calibration plot for simulated data $(\mathrm{n}=500)$. The green triangles denote the mean predicted and observed event probabilities for patients grouped into tenths using deciles. The gray dashed line denotes perfect calibration. A smoothing curve (blue dotted line) and the calibration curve (red solid line) are also overlaid. The distribution of calculated predicted probabilities is overlaid along the horizontal axis. A subset of various statistics useful for validating the model are also shown. This figure was generated using standard statistical software: the rms package for $\mathrm{R}$ version 3.1.2 (R Foundation for Statistical Computing, Vienna, Austria). More details have been provided by Harrell. ${ }^{38,46}$ Code to reproduce this plot is given in Appendix 1. ROC, Receiver operating characteristic curve.

\section{MODEL UPDATING}

A natural extension to the validation of a CRPM is the concept of updating an existing model. This might involve exploring whether a new biomarker improves a model (eg, using net reclassification improvement measures),${ }^{42}$ recalibrating a model, ${ }^{43}$ and, more recently, assessing whether multiple models can be combined to provide a more accurate prediction (eg, meta-models and model averaging). ${ }^{44}$ This expanding research area is especially important in the current era of personalized medicine. ${ }^{45}$

\section{CONCLUSIONS}

External validation of CRPMs is necessary to demonstrate their predictive accuracy. Available models likely have been validated internally; however, using them in different settings, locations, and populations can result in relatively poor performance. CRPMs that have been overfitted during development also will often fail to generalize to the external validation sample. Calibration and discrimination must be measured to establish validity. Multiple statistical approaches to interrogate the calibration are available, with the general recognition that the ubiquitous HosmerLemeshow test has limited utility. Execution of a rigorous
CRPM validation study rests in proper study design, application of suitable statistical methods, and transparent reporting.

\section{Conflict of Interest Statement}

Authors have nothing to disclose with regard to commercial support.

\section{References}

1. Moons KG, Royston P, Vergouwe Y, Grobbee DE, Altman DG. Prognosis and prognostic research: what, why, and how? BMJ. 2009;338:b375.

2. Leon MB, Smith CR, Mack M, Miller DC, Moses JW, Svensson LG, et al. Transcatheter aortic-valve implantation for aortic stenosis in patients who cannot undergo surgery. N Engl J Med. 2010;363:1597-607.

3. Bridgewater B, Hickey GL, Cooper G, Deanfield J, Roxburgh J. Publishing cardiac surgery mortality rates: lessons for other specialties. BMJ. 2013;346:f1139.

4. Altman DG, Vergouwe Y, Royston P, Moons KG. Prognosis and prognostic research: validating a prognostic model. BMJ. 2009;338:b605.

5. Harrell FE Jr, Lee KL, Mark DB. Multivariable prognostic models: issues in developing models, evaluating assumptions and adequacy, and measuring and reducing errors. Stat Med. 1996;15:361-87.

6. Steyerberg EW. Clinical Prediction Models: A Practical Approach to Development, Validation, and Updating. New York: Springer; 2009.

7. Nilsson J, Ohlsson M, Thulin L, Höglund P, Nashef SA, Brandt J. Risk factor identification and mortality prediction in cardiac surgery using artificial neural networks. J Thorac Cardiovasc Surg. 2006;132:12-9.

8. Moons KG, Altman DG, Vergouwe Y, Royston P. Prognosis and prognostic research: application and impact of prognostic models in clinical practice. BMJ. 2009;338:b606.

9. Rufibach K. Use of Brier score to assess binary predictions. J Clin Epidemiol. 2010;63:938-9.

10. Grant SW, Hickey GL, Dimarakis I, Trivedi U, Bryan A, Treasure T, et al. How does EuroSCORE II perform in UK cardiac surgery; an analysis of 23,740 patients from the Society for Cardiothoracic Surgery in Great Britain and Ireland National Database. Heart. 2012;98:1568-72.

11. Grant SW, Hickey GL, Dimarakis I, Cooper G, Jenkins DP, Uppal R, et al. Performance of the EuroSCORE models in emergency cardiac surgery. Circ Cardiovasc Qual Outcomes. 2013;6:178-85.

12. Knol MJ, Janssen KJ, Donders AR, Egberts AC, Heerdink ER, Grobbee DE, et al. Unpredictable bias when using the missing indicator method or complete case analysis for missing confounder values: an empirical example. J Clin Epidemiol. 2010;63:728-36.

13. Marshall A, Altman DG, Holder RL, Royston P. Combining estimates of interest in prognostic modelling studies after multiple imputation: current practice and guidelines. BMC Med Res Methodol. 2009;9:57.

14. Janssen KJ, Vergouwe Y, Donders AR, Harrell FE Jr, Chen Q, Grobbee DE, et al. Dealing with missing predictor values when applying clinical prediction models. Clin Chem. 2009;55:994-1001.

15. Vergouwe Y, Steyerberg EW, Eijkemans MJ, Habbema JD. Substantial effective sample sizes were required for external validation studies of predictive logistic regression models. J Clin Epidemiol. 2005;58:475-83.

16. Collins GS, Ogundimu EO, Altman DG. Sample size considerations for the external validation of a multivariable prognostic model: a resampling study. Stat Med. 2016;35:214-26.

17. Roques F, Michel P, Goldstone AR, Nashef SA. The logistic EuroSCORE. Eur Heart J. 2003;24:881-2.

18. Shahian DM, O'Brien SM, Filardo G, Ferraris VA, Haan CK, Rich JB, et al. The Society of Thoracic Surgeons 2008 cardiac surgery risk models, part 1: coronary artery bypass grafting surgery. Ann Thorac Surg. 2009;88(1 Suppl):S2-22.

19. O'Brien SM, Shahian DM, Filardo G, Ferraris VA, Haan CK, Rich JB, et al. The Society of Thoracic Surgeons 2008 cardiac surgery risk models, part 2: isolated valve surgery. Ann Thorac Surg. 2009;88(1 Suppl):S23-42.

20. Shahian DM, O'Brien SM, Filardo G, Ferraris VA, Haan CK, Rich JB, et al. The Society of Thoracic Surgeons 2008 cardiac surgery risk models, part 3: valve plus coronary artery bypass grafting surgery. Ann Thorac Surg. 2009;88(1 Suppl): S43-62.

21. Jacobs JP, Mavroudis C, Jacobs ML, Maruszewski B, Tchervenkov CI, LacourGayet FG, et al. What is operative mortality? Defining death in a surgical registry database: a report of the STS Congenital Database Taskforce and the Joint 
EACTS-STS Congenital Database Committee. Ann Thorac Surg. 2006;81: 1937-41.

22. Swinkels BM, Plokker HW. Evaluating operative mortality of cardiac surgery: first define operative mortality. Neth Heart J. 2010;18:344-5.

23. Nashef SA, Roques F, Sharples LD, Nilsson J, Smith C, Goldstone AR, et al. EuroSCORE II. Eur J Cardiothorac Surg. 2012;41:734-44.

24. Siregar S, Groenwold RH, de Mol BA, Speekenbrink RG, Versteegh MI, Brandon Bravo Bruinsma GJ, et al. Evaluation of cardiac surgery mortality rates: 30-day mortality or longer follow-up? Eur J Cardiothorac Surg. 2013; 44:875-83.

25. Hickey GL, Grant SW, Cosgriff R, Dimarakis I, Pagano D, Kappetein AP, et al. Clinical registries: governance, management, analysis and applications. Eur J Cardiothorac Surg. 2013;44:605-14.

26. Hickey GL, Grant SW, Murphy GJ, Bhabra M, Pagano D, McAllister K, et al. Dynamic trends in cardiac surgery: why the logistic EuroSCORE is no longer suitable for contemporary cardiac surgery and implications for future risk models. Eur J Cardiothorac Surg. 2013;43:1146-52.

27. Hickey GL, Grant SW, Caiado C, Kendall S, Dunning J, Poullis M, et al. Dynamic prediction modeling approaches for cardiac surgery. Circ Cardiovasc Qual Outcomes. 2013;6:649-58.

28. Schulz KF, Altman DG, Moher D. CONSORT 2010 Statement: updated guidelines for reporting parallel group randomised trials. Trials. 2010;11:32.

29. Moher D, Liberati A, Tetzlaff J, Altman DG. Preferred reporting items for systematic reviews and meta-analyses: the PRISMA statement. PLoS Med. 2009;6:e1000097.

30. Collins GS, Reitsma JB, Altman DG, Moons KG. Transparent reporting of a multivariable prediction model for individual prognosis or diagnosis (TRIPOD): the TRIPOD statement. Circulation. 2015;131:211-9.

31. Moons KG, Altman DG, Reitsma JB, Ioannidis JP, Macaskill P, Steyerberg EW, et al. Transparent reporting of a multivariable prediction model for individual prognosis or diagnosis (TRIPOD): explanation and elaboration. Ann Intern Med. 2015;162:W1-73.

32. Kramer AA, Zimmerman JE. Assessing the calibration of mortality benchmarks in critical care: the Hosmer-Lemeshow test revisited. Crit Care Med. 2007;35: 2052-6.

33. Marcin JP, Romano PS. Size matters to a model's fit. Crit Care Med. 2007;35: 2212-3.

34. Paul P, Pennell ML, Lemeshow S. Standardizing the power of the HosmerLemeshow goodness of fit test in large data sets. Stat Med. 2013;32:67-80.

35. Bertolini G, D'Amico R, Nardi D, Tinazzi A, Apolone G. One model, several results: the paradox of the Hosmer-Lemeshow goodness-of-fit test for the logistic regression model. J Epidemiol Biostat. 2000;5:251-3.

36. Hosmer DW, Lemeshow S. Applied Logistic Regression. 2nd ed. New York: Wiley; 2000.

37. Austin PC, Steyerberg EW. Graphical assessment of internal and external calibration of logistic regression models by using loess smoothers. Stat Med. 2014;33:517-35.

38. Harrell FE Jr. Regression Modeling Strategies: With Applications to Linear Models, Logistic Regression, and Survival Analysis. New York: Springer; 2001.

39. Cox DR. Two further applications of a model for binary regression. Biometrika. 1958;45:562-5.

40. Spiegelhalter DJ. Probabilistic prediction in patient management and clinical trials. Stat Med. 1986;5:421-33.
41. Stallard N. Simple tests for the external validation of mortality prediction scores Stat Med. 2009;28:377-88.

42. Pencina MJ, D'Agostino RB Sr, D'Agostino RB Jr, Vasan RS. Evaluating the added predictive ability of a new marker: from area under the ROC curve to reclassification and beyond. Stat Med. 2008;27:157-72.

43. Steyerberg EW, Borsboom GJ, van Houwelingen HC, Eijkemans MJ, Habbema JD. Validation and updating of predictive logistic regression models: a study on sample size and shrinkage. Stat Med. 2004;23:2567-86.

44. Debray TP, Koffijberg H, Nieboer D, Vergouwe Y, Steyerberg EW, Moons KG Meta-analysis and aggregation of multiple published prediction models. Stat Med. 2014;33:2341-62.

45. Su T-L, Jaki TF, Hickey G, Buchan I, Sperrin M. A review of statistical updating methods for clinical prediction models. Stat Methods Med Res. 2015 In press.

46. Harrell FE Jr. rms: regression modeling strategies. Available at: http://cran.rproject.org/package =rms. Accessed April 19, 2016.

Key Words: risk prediction model, external validation, calibration, discrimination, statistics

\section{APPENDIX 1. R CODE TO PRODUCE FIGURE 1}

\# If 'rms' package not installed, run command

\# install.packages("rms")

library(rms)

\#\# Simulate fake data:

\#\# $\mathrm{y}=$ binary outcome

\#\# $\mathrm{x} 1, \mathrm{x} 2, \mathrm{x} 3=$ covariates in the risk model

\#\# $\mathrm{n}=$ sample size

set.seed(1)

$\mathrm{n}<-1000$ \# 500 development +500 validation

$\mathrm{x} 1<-\operatorname{runif}(\mathrm{n})$ \# covariate 1

$\mathrm{x} 2<-\operatorname{runif}(\mathrm{n})$ \# covariate 2

x $3<-$ runif(n) \# covariate 3

$\operatorname{logit}<--5+0.5 * \mathrm{x} 1+2 * \mathrm{x} 2+3.5 * \mathrm{x} 3$

$\mathrm{P}<-1 /(1+\exp (-\operatorname{logit}))$

$\mathrm{y}<$ - ifelse(runif(n) $<=\mathrm{P}, 1,0)$ \# outcomes

$\mathrm{d}<$ - data.frame(x1, x2, x3, y) \# combined dataset

\#\# Fit a risk prediction model to first half of the data

$\mathrm{f}<-\operatorname{lrm}(\mathrm{y} \sim \mathrm{x} 1+\mathrm{x} 2+\mathrm{x} 3$, subset $=1: 500)$

\#\# Use model to get predictions for second half of data pred.logit <- $\operatorname{predict}(f, \mathrm{~d}[501: 1000]$,

phat $<-1 /(1+\exp (-$ pred.logit $))$

\#\# Validate prediction

val.prob(phat, $\mathrm{y}[501: 1000], \mathrm{g}=10$, riskdist $=$ "predicted") 